\title{
ABORDAGEM ORTOPÉDICA DAS DEFORMIDADES PECTUS: 32 ANOS DE ESTUDOS
}

\author{
ORTHOPAEDIC APPROACH TO PECTUS DEFORMITIES: \\ 32 YEARS OF STUDIES
}

Sydney Abrão Haje', Davi de Podestá Haje²

\section{RESUMO}

Os autores resumem experiência de 32 anos no estudo e na abordagem não cirúrgica das deformidades pectus carinatum e pectus excavatum. Dados de 4.012 pacientes portadores de deformidades pectus foram coletados de 1977 a janeiro de 2009, permitindo avaliar sua etiologia, patogênese e tratamento. Distúrbios de crescimento dos ossos e cartilagens da parede torácica anterior foram detectados em exames de imagem. A hereditariedade e fatores biomecânicos, como distúrbios respiratórios e escolioses, mostraram-se presentes em mais de $40 \%$ dos pacientes. O método dinâmico de remodelação do tórax - órteses compressivas simultaneamente à prática de exercícios - foi indicado em 2.453 pacientes. Tratamento concomitante com colete inclinado foi instituído em 11 portadores de escoliose entre $20^{\circ}$ e $52^{\circ}$. Dos portadores de pectus que tiveram o tratamento indicado, 1.717 retornaram para reavaliação: 1.632 crianças e adolescentes e 85 adultos. Bons resultados foram observados em $60,6 \%$ das crianças e adolescentes e em $27 \%$ dos adultos tratados. Nenhum caso de escoliose apresentou piora da curva e um caso de $52^{\circ}$ teve melhora de $20^{\circ}$ com o tratamento. Distúrbios no crescimento do esterno e arcos costais, bem como os fatores biomecânicos que compõem a patogênese das deformidades pectus, demonstram como essas deformidades estão correlacionadas com a ortopedia. Avaliação apropriada da parede torácica anterior e tratamento concomitante com colete inclinado são recomendados na presença de escoliose. O método dinâmico de remodelação do tórax exige um protocolo de condutas médicas para que o tratamento tenha êxito.

Descritores - Tórax em funil; Parede torácica; Remodelação óssea; Aparelhos ortopédicos

\section{ABSTRACT}

The authors summarize a 32-year experience in the study and in the non-operative approach of pectus carinatum and pectus excavatum. Data of 4,012 patients with pectus deformities were collected from 1977 to January 2009, allowing evaluation on the etiology, pathogenesis and treatment of these deformities. Growth disturbances of anterior chest wall bones and cartilages were detected in imaging studies. Heredity, and biomechanical factors, like respiratory disturbances and scoliosis were noticed in more than $40 \%$ of the patients. The method of dynamic remodeling of the thorax - compressive orthoses simultaneously to exercises practice - was indicated in 2453 patients. Concomitant treatment with bending brace was provided in patients with $20^{\circ}$ to $52^{\circ}$ scoliosis. Of pectus patients with treatment indication, 1717 returned for re-evaluation: 1632 children and adolescents and 85 adults. Good results were seen in $60.6 \%$ of children and adolescents and in 27\% of adults treated. No scoliosis patient presented curve worsening, and a case of $52^{\circ}$ presented an improvement of $20^{\circ}$ in the scoliosis with the treatment. Disturbances in the growth of the sternum and costal arches, as well as biomechanical factors related to the pathogenesis of pectus deformities, demonstrate how these deformities are correlated to orthopaedics. Appropriate evaluation of the anterior chest wall and concomitant treatment with bending brace are recommended in the presence of scoliosis. The dynamic remodeling method of the thorax requires a protocol of medical actions for a successful treatment.

Keywords - Funnel chest; Thoracic wall. Bone remodeling; Orthotic devices

1 - Ortopedista Pediátrico e Fisiatra do Centro Clínico Orthopectus e do Hospital Regional da Asa Norte.

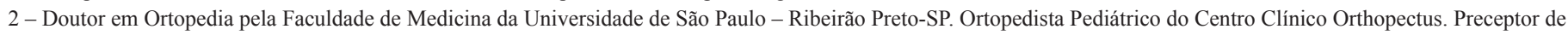
Ortopedia Pediátrica e Pé Adulto do Hospital de Base do Distrito Federal - Brasília-DF.

Correspondência: SMHN Quadra 2 Bloco A Edifício de Clínicas, salas 804-806, Brasília-DF, Brasil. CEP 70710-904. Telefax: (61) $3425-1408$.

E-mail: orthohaje@orthopectus.com.br 


\section{INTRODUÇÃO}

Prevalência e aspectos psicológicos - As deformidades da parede torácica anterior, designadas universalmente como deformidades pectus, são observadas com frequência na prática médica. Garcia et $a l^{(1)}$ relatam um caso de pectus excavatum para cada 300 nascidos vivos e Haje et $a l^{(2)}$ descrevem um caso de pectus para cada 100 escolares examinados. Entretanto, essas deformidades são geralmente escondidas pelos pacientes devido a problemas psicológicos, tornando-as desconhecidas. $\mathrm{O}$ infortúnio de se portar uma deformidade pectus influencia todas as áreas da vida. De acordo com Einsiedel e Clausner, os efeitos psicológicos são maiores após os 11 anos de idade, quando se observam sentimentos de constrangimento, ansiedade social, vergonha, limitada capacidade para atividades e comunicação, negativismo, intolerância, frustração e até mesmo reações depressivas $^{(3)}$. Por outro lado, o suporte médico adequado ao portador de uma deformidade pectus contribui para o restabelecimento da saúde mental do paciente, reabilitando-o ao convívio social normal e trazendo alívio para sua família ${ }^{(4)}$. Diante do grande sofrimento psíquico que as deformidades muitas vezes acarretam a seus portadores, faz-se necessário que o médico, especialmente o ortopedista, tenha conhecimentos sobre a formação dos ossos e cartilagens da parede torácica anterior, a etiologia, a patogênese e as opções de tratamento dos diversos tipos de deformidade pectus.

Anatomopatologia e formação embriológica do esterno e arcos costais - Muitos autores já utilizaram o termo "suturas" para definir linhas radiotransparentes no esterno em crescimento ${ }^{(5-13)}$, mas é importante destacar que suturas existem apenas no crânio e que a formação e o crescimento da parede torácica anterior são endocondrais $^{(14-16)}$; há a presença de placas de crescimento cartilaginosas entre os segmentos do esterno em crescimento e nas junções costocondrais ${ }^{(17-22)}$. A formação do esterno ocorre a partir de duas bandas longitudinais de mesoderma, provenientes da região dos ombros, que se unem no sentido craniocaudal à medida que acontece um processo de condrificação. Cartilagens costais provenientes da coluna vertebral se fundem ao esterno em formação e placas cartilaginosas de crescimento se desenvolvem nesses locais de encontro das cartilagens costais com o esterno, sendo responsáveis pelo crescimento esternal ${ }^{(23,24)}$. Placas de crescimento costais também existem na junção costocondral dos arcos costais. $\mathrm{Na}$ abordagem dos ossos longos das crianças e adolescentes, o cirurgião ortopédi- co evita lesar placas cartilaginosas de crescimento, pois sabe que tal lesão acarretará uma deformidade no futuro. Tal cuidado, entretanto, parece inexistir em relação ao tórax, tendo em vista que, dentre as dezenas de técnicas cirúrgicas descritas para tratar as deformidades pectus, não existe aquela que mencione atenção às placas de crescimento esternais e costais ${ }^{(19)}$.

\section{ETIOLOGIA E PATOGÊNESE}

Distúrbios no crescimento dos ossos e das cartilagens da parede torácica anterior são descritos na literatura como agente etiológico ${ }^{(21,22)}$. Assim sendo, as deformidades pectus têm tendência para progressão natural no pico de crescimento da adolescência ${ }^{(23,24)}$. Fatores biomecânicos como distúrbios respiratórios, escolioses e cifoses contribuem para a patogênese das deformidades pectus $^{(22-24)}$.

Casuística dos autores e classificação (tipos de deformidades pectus) - De 1977 a janeiro de 2009, foram examinados 4.012 pacientes portadores de deformidades pectus, sendo $73 \%$ do sexo masculino e $27 \%$ do feminino. Conforme previamente descrito, as deformidades foram classificadas de acordo com a localização anatômica da área protrusa ou da área deprimida ${ }^{(19,23,25)}$. Sempre que a deformidade predominante foi uma protrusão na região esternal, junção condroesternal ou cartilagem costal adjacente ao esterno, ela foi classificada como pectus carinatum. A presença exclusiva de uma depressão esternal caracterizou o pectus excavatum. Classificamos o pectus carinatum em três tipos básicos, conforme a localização anatômica do ápice da protrusão: pectus carinatum inferior (PCI), pectus carinatum lateral (PCL) e pectus carinatum superior (PCS). O pectus excavatum foi classificado, de acordo com a extensão da depressão, em tipos localizado (PEL) e amplo (PEA). Além dos tipos básicos de pectus, tipos mistos de deformidades também foram detectados, sendo o diagnóstico firmado sempre pela deformidade predominante ${ }^{(23,24)}$. Nesses casos se deve anotar, em adição ao diagnóstico principal, a(s) deformidade(s) secundária(s), como, por exemplo: PCS + PEL + PCL esquerdo; PCL direito + PEL; PEA + PCL esquerdo, PCI+PCL direito, etc. Saliências dos rebordos costais na parte inferior da parede torácica anterior, quando presentes, foram registradas para acompanhamento adequado e definição terapêutica. $\mathrm{O}$ pectus carinatum mostrou-se mais frequente em nossa casuística, estando presente em 3.111 pacientes (78\%), com o diagnóstico de PCI sendo feito em 1.785 (45\%), de PCL em 1.126 (28\%) 
e de PCS em 200 (5\%). Já o pectus excavatum mostrou-se presente em 901 pacientes (22\%), sendo o diagnóstico de PEL feito em 529 (13\%) e o de PEA em 372 (9\%).

Exames de imagem para análise etiológica - Radiografias do esterno em perfil/oblíqua foram rotineiramente realizadas e analisadas conforme critérios descritos por Haje et $a l^{(22)}$. Tomografia computadorizada (TC) multislice da parede torácica anterior foi feita em 94 pacientes e a reconstrução coronal utilizada para melhor interpretar possíveis distúrbios envolvendo a formação, o crescimento e o desenvolvimento do esterno e das cartilagens costais, ou seja, para análise etiológica. A reconstrução coronal da TC multislice permitiu melhor visualização das placas cartilaginosas do esterno e da morfologia do corpo esternal do que as radiografias, possibilitando ainda a visualização das cartilagens costais e facilitando a interpretação de distúrbios que envolvam a formação, o crescimento e o desenvolvimento do esterno e arcos costais. Nesse exame, puderam ser detectadas imagens sugestivas de distúrbios na formação, como: 1) ossificação e fusão do xifoide ao corpo; 2) irregularidade látero-lateral na morfologia do corpo esternal; 3) assimetria das cartilagens costais nos hemitórax; 4) ossificação e fusão do manúbrio ao corpo; 5) irregularidade nas placas cartilaginosas de crescimento do esterno; 6) fusão incompleta das bandas longitudinais de origem embrionária do esterno; 7) fusão de todos os segmentos do corpo esternal; 8) impressão de alargamento do corpo esternal. Na Figura 1 são mostrados esses aspectos em dois portadores de pectus idiopático - forma de pectus esta detalhada a seguir.

Formas de ocorrência - Os autores investigaram em cada paciente de sua casuística eventual doença esquelética concomitante e procedimento operatório prévio sobre o esterno. Três formas de ocorrência foram detectadas: a patológica, a iatrogênica e a idiopática. A patológica, que ocorre na presença de doenças que têm associação com distúrbios de crescimento de maneira geral, como síndrome de Marfan, displasias ósseas e osteogenesis imperfecta, foi diagnosticada em 11 pacientes de nossa casuística $(0,3 \%)$. A iatrogênica, devido à lesão e ao desarranjo anatômico das placas de crescimento esternais, foi detectada em 22 pacientes $(0,5 \%$ do total de nossos casos), sendo 21 casos após procedimento operatório cardíaco e um caso após punção esternal neonatal. A forma idiopática, isso é, aquela que acomete pessoas que têm boa condição de saúde geral, foi observada em 3.979 dos pacientes estudados $(99,2 \%)$.

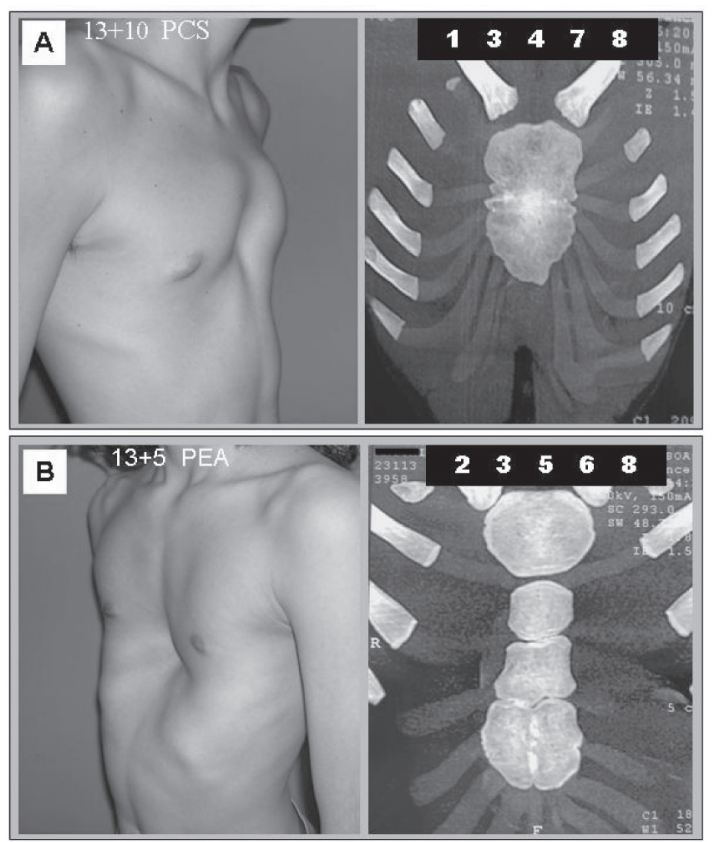

Figura 1 - Exemplos de aspectos encontrados na reconstrução coronal de TC multislice do tórax em adolescentes portadores de $(A)$ pectus carinatum superior (PCS) e (B) pectus excavatum amplo (PEA), ambos com 13 anos de idade. Os aspectos 3) assimetria das cartilagens costais nos hemitórax e 8) impressão de alargamento do corpo esternal são encontrados em ambos, enquanto os aspectos 1) ossificação e fusão do xifoide ao corpo, 4) ossificação e fusão do manúbrio ao corpo e 7) fusão de todos os segmentos do corpo esternal são visíveis apenas em (A) e os aspectos 2) irregularidade látero-lateral na morfologia do corpo esternal, 5) irregularidade nas placas cartilaginosas de crescimento do esterno, e 6) fusão incompleta das bandas longitudinais de origem embrionária do esterno são percebidos apenas em (B).

História clínica e exames para análise da patogênese - Os pacientes e/ou responsáveis foram questionados quanto à presença de outros casos na família (hereditariedade) e fatores biomecânicos que pudessem influenciar o crescimento e o desenvolvimento da parede torácica anterior, como distúrbios respiratórios (asma, pneumonia, hipertrofia de adenoide, sinusite e rinite alérgica). Exames físicos e, quando necessários, radiológicos, foram rotineiramente feitos para pesquisa de desvios da coluna vertebral (cifoses torácicas exacerbadas e/ou escolioses). $\mathrm{O}$ relato de outros casos de pectus na família aconteceu em $47 \%$ dos pacientes. Distúrbios respiratórios prévios, como asma, pneumonia, hipertrofia de adenoide, sinusite e rinite alérgica, foram relatados por $50 \%$ dos pacientes. Exacerbação da cifose torácica mostrou-se presente em 14\% dos casos. Escoliose de $5^{\circ}$ a $19^{\circ}$ foi detectada em 1.685 pacientes ( $42 \%$ dos casos) e entre $20^{\circ}$ e $52^{\circ}$ em 52 pacientes ( $1,3 \%$ dos casos). 


\section{TRATAMENTO CONSERVADOR / MÉTODO DR}

Tratamento com órteses compressivas dinâmicas (CDT - compressor dinâmico de tórax) foi inicialmente descrito em 1979 para o pectus carinatum ${ }^{(11,13,26)}$. Órteses compressivas com velcros ou fechos nas laterais têm sido descritas como opções terapêuticas conservadoras efetivas para crianças com tipos flexíveis de pectus carinatum $^{(27-31)}$. Tratamento com órteses CDT - que têm parafusos nas laterais, em vez de velcros ou fechos, e possibilitam compressão gradual de áreas protrusas - passou a ser descrito por Haje et al não só para o pectus carinatum, mas também para o pectus excavatum, a partir de $1992^{(19,23,32)}$. Em 2006, os autores sintetizaram a descrição de seu método com a publicação do termo Dynamic Remodeling (DR) method - método dinâmico de remodelação - ou método DR, para designar o uso de órteses CDT simultaneamente à prática de exercícios que promovam aumento da pressão intratorácica ${ }^{(33)}$. Tal método implica equilíbrio de forças sobre o tórax: enquanto $\mathrm{a}(\mathrm{s})$ órtese(s) exerce $(\mathrm{m})$ pressão externa dinâmica sobre áreas protrusas ou salientes, a execução de exercícios, simultaneamente ao uso de uma ou duas órteses CDT, promove pressão interna sobre áreas deprimidas, proporcionando a remodelação da caixa torácica como um todo.

Tratamento conservador / método DR - Dos 4.012 pacientes examinados, 2.453 tiveram indicação para início do tratamento pelo método DR. Desses, 2.278 eram crianças e adolescentes, ou seja, pacientes com idade máxima de 19 anos, e 175 eram adultos. A prescrição de órteses para adultos aconteceu apenas para pacientes extremamente incomodados com o aspecto estético de seu tórax e foi sempre precedida de explicação sobre as dificuldades do tratamento na vida adulta. Já para indivíduos em crescimento, o critério foi o seguinte: na infância, para portadores de PCS (tipo de deformidade rígida desde a infância); na pré-adolescência, para portadores de PEL e PEA; e na adolescência, para pacientes portadores de PCI e PCL (tipos de deformidades mais flexíveis). Na infância, portadores de PCI, PCL, PEL e PEA apenas receberam prescrição de órtese na presença de história de distúrbio respiratório frequente ou, eventualmente, quando a deformidade se apresentou de moderada a severa ou progressiva. A não prescrição de órteses aconteceu para deformidades discretas ou para crianças muito jovens, portadoras de PCI, PCL, PEL e PEA, sem história de distúrbio respiratório, sendo, nes- ses casos, indicados apenas documentação fotográfica e acompanhamento.

Fotografias clínicas do tórax de cada paciente foram rotineiramente realizadas, sempre de um mesmo ângulo oblíquo-lateral, para acompanhamento. Para avaliação dos resultados do tratamento, classificamos a melhora observada, baseados na análise das fotos, em índices de melhora (IM), sendo (3) igual a melhora boa ou excelente, (2) moderada, (1) discreta e (0) nenhuma.

Melhora observada com o método DR - Dos 2.453 pacientes que tiveram indicação para início do tratamento pelo método dinâmico de remodelação (2.278 crianças/adolescentes e 175 adultos), 1.717 retornaram para reavaliação: 1.632 crianças e adolescentes e 85 adultos. As crianças e adolescentes compuseram, portanto, 95\% dos pacientes que aderiram ao tratamento, enquanto os adultos, apenas $5 \%$.

Das 2.278 crianças e adolescentes que iniciaram tratamento, 1.632 (71,6\% das que tiveram indicação para tal) retornaram e foram acompanhadas por tempo de seguimento médio de um ano e sete meses (mínimo de um mês, máximo de 18 anos e cinco meses). A Tabela 1 mostra os índices de melhora obtidos para crianças e adolescentes com o método DR em cada tipo de deformidade, em valores absolutos e com os percentuais em cada grupo. O percentual geral de crianças e adolescentes com melhora (3) e (2) foi de $60,6 \%$.

Dos 175 adultos que iniciaram tratamento, 85 (48,6\% entre aqueles que tiveram indicação para tal) retornaram para reavaliação em tempo de seguimento médio de um ano (mínimo um mês, máximo quatro anos e cinco me-

Tabela 1 - Índice de melhora (IM) com tratamento pelo método dinâmico de remodelação do tórax (método DR) em crianças e adolescentes. Distribuição dos 1.632 pacientes que retornaram para reavaliação do tratamento de acordo com o tipo de deformidade. O número de pacientes e os IM obtidos para cada tipo de deformidade são expressos pelos números menores, e os respectivos percentuais, pelos números maiores, em negrito. Pacientes com IM 3 e IM 2 (totais em destaque) compuseram $60,6 \%$ dos casos.

\begin{tabular}{l|l|l|l|l|l}
\hline \multicolumn{2}{l|}{$\begin{array}{l}\text { Índice de melhora } \\
\text { 454 PCL } \\
781 \text { PCI }\end{array}$} & 69 PCS & 178 PEL & 150 PEA & 1632 Total \\
\hline IM 3 & $\begin{array}{l}14231,3 \% \\
38849,7 \%\end{array}$ & $57,3 \%$ & $116,2 \%$ & $21,3 \%$ & $54833,6 \%$ \\
\hline IM 2 & $13930,6 \%$ & $1521.7 \%$ & $4022.5 \%$ & $3020.0 \%$ & $44027,0 \%$ \\
& $21627,7 \%$ & & & & \\
\hline IM 1 & $8719,2 \%$ & $2942,0 \%$ & $8246,0 \%$ & $7952,7 \%$ & $36622,4 \%$ \\
& $8911,4 \%$ & & & & \\
\hline IM 0 & $8618,9 \%$ & $2029,0 \%$ & $4525,3 \%$ & $3926,0 \%$ & $27817,0 \%$ \\
& $8811,2 \%$ & & & & \\
\hline
\end{tabular}


ses). A tabela 2 apresenta os índices de melhora obtidos para adultos com o método DR em cada tipo de deformidade, em valores absolutos e com os percentuais em cada grupo. O percentual geral de adultos com melhora (3) e (2) foi de $27 \%$.

Tabela 2 - Índice de melhora (IM) com tratamento pelo método dinâmico de remodelação (método DR) em adultos. Distribuição dos 85 pacientes que retornaram para reavaliação do tratamento de acordo com o tipo de deformidade. O número de pacientes e os IM obtidos para cada tipo de deformidade são expressos pelos números menores, e os respectivos percentuais, pelos números maiores, em negrito. Pacientes com IM 3 e IM 2 (totais em destaque) compuseram $27 \%$ dos casos.

\begin{tabular}{c|c|c|c|c|c|c}
\hline $\begin{array}{c}\text { Índice de } \\
\text { melhora }\end{array}$ & $23 \mathrm{PCl}$ & $15 \mathrm{PCL}$ & 4 PCS & 27 PEL & $\begin{array}{c}16 \\
\text { PEA }\end{array}$ & $\begin{array}{c}85 \\
\text { Total }\end{array}$ \\
\hline IM 3 & $521,7 \%$ & - & - & $13,7 \%$ & - & $67,0 \%$ \\
\hline IM 2 & $626,1 \%$ & $533,3 \%$ & $125,0 \%$ & $414,8 \%$ & $16,2 \%$ & $1720,0 \%$ \\
\hline IM 1 & $626,1 \%$ & $746,7 \%$ & $375,0 \%$ & $1451,9 \%$ & $743,8 \%$ & $3743,6 \%$ \\
\hline IM 0 & $626,1 \%$ & $320,0 \%$ & - & $829,6 \%$ & $850,0 \%$ & $2529.4 \%$ \\
\hline
\end{tabular}

O tempo de seguimento mínimo de um mês foi adotado porque, com esse tempo, muitas vezes já se observa melhora ou até mesmo hipercorreção ${ }^{(33)}$ para carinatum flexível, mas, isso não significa que o tratamento deva ser interrompido. Quanto menor o tempo de tratamento, maior a probabilidade de recorrência, sendo o acompanhamento médico fundamental para a adequada remodelação da parede torácica anterior. Se saliências de rebordos costais se formam ou se exacerbam com a compressão de uma protrusão esternal, a adição de órtese CDT II deve acontecer ao tratamento. A retirada(s) da(s) órtese(s) deve ser feita de maneira gradual e a alta médica só deve acontecer após certeza de estabilização da correção. Exemplo de paciente com pectus excavatum tratada com sucesso pode ser visto na figura 2 .

Irritação cutânea nos locais onde as almofadas do CDT fazem pressão foi observada, em maior ou menor intensidade, em todos pacientes que utilizaram a órtese de maneira correta. Tal irritação pode ser minimizada com orientação adequada em acompanhamento médico periódico e desaparece naturalmente com a interrupção ou término do tratamento. Hipercorreção da deformidade original foi detectada em 61 casos, complicação essa corrigida em todos os casos por meio de condutas individualizadas, descritas em publicação prévia ${ }^{(33)}$.

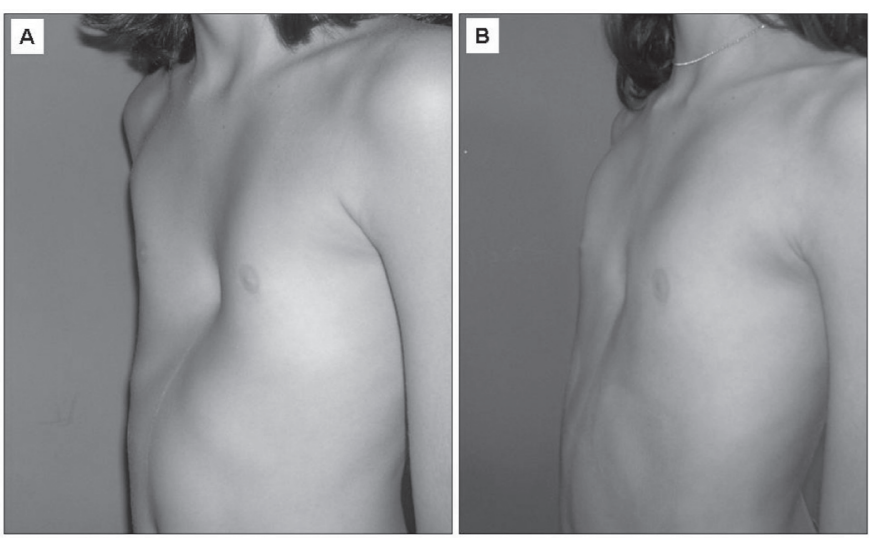

Figura 2 - Pré-adolescente do sexo feminino portadora de pectus excavatum localizado, aos 10 anos de idade (A) e um ano após tratamento pelo método DR (B). A melhora observada (IM 3) denota a assiduidade da paciente no uso da órtese CDT II e na execução dos exercícios, que relatou realizar regularmente.

De 52 pacientes portadores de deformidades pectus e escoliose entre $20^{\circ}$ e $52^{\circ}, 11$ receberam também tratamento concomitante com colete inclinado de Brasília ou $\mathrm{CIB}^{(34)}$. Nesses casos, a(s) órtese(s) CDT(s) foi/foram utilizada(s) por quatro horas diárias, o programa de exercícios do método DR feito em uso das mesmas, incluindo-se alongamentos da coluna na vertical e flexões laterais em maior número de vezes para o lado que apresentava melhora da curva nas inclinações laterais de radiografias pré-tratamento, e a utilização do CIB feita pelo resto do dia (Figura 3). Dos 11 pacientes portadores de deformidades pectus e escoliose de valor angular entre $20^{\circ}$ e $52^{\circ}$, que receberam, além do tratamento pelo método DR, também tratamento concomitante com colete inclinado de Brasília ou CIB, oito apresentaram melhora igual ou superior a $5^{\circ}$ na curva escoliótica, sendo a melhora em um dos casos igual a $20^{\circ}$ (Figura 4 ).
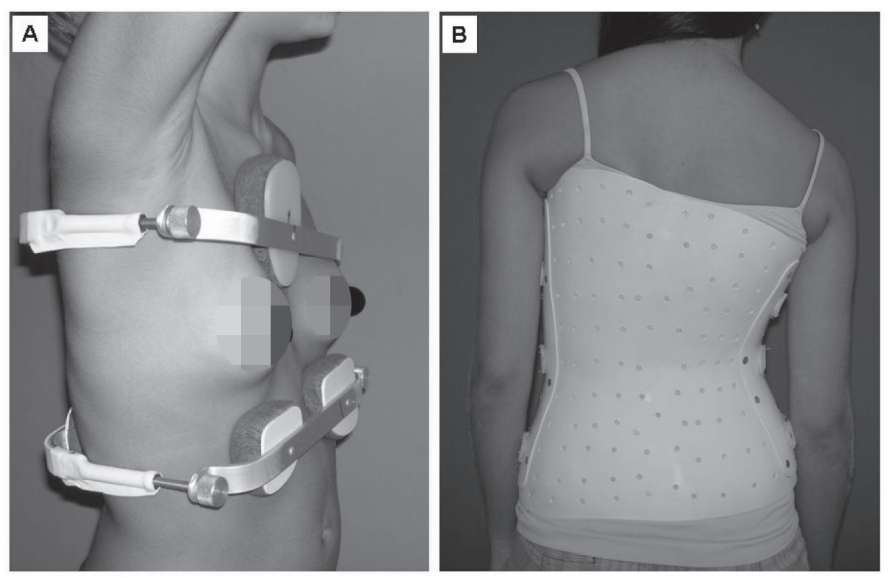

Figura 3 - Paciente utilizando órteses CDT e colete inclinado de Brasília $(\mathrm{CIB})(34)$ para tratamento concomitante de deformidade pectus e escoliose. 

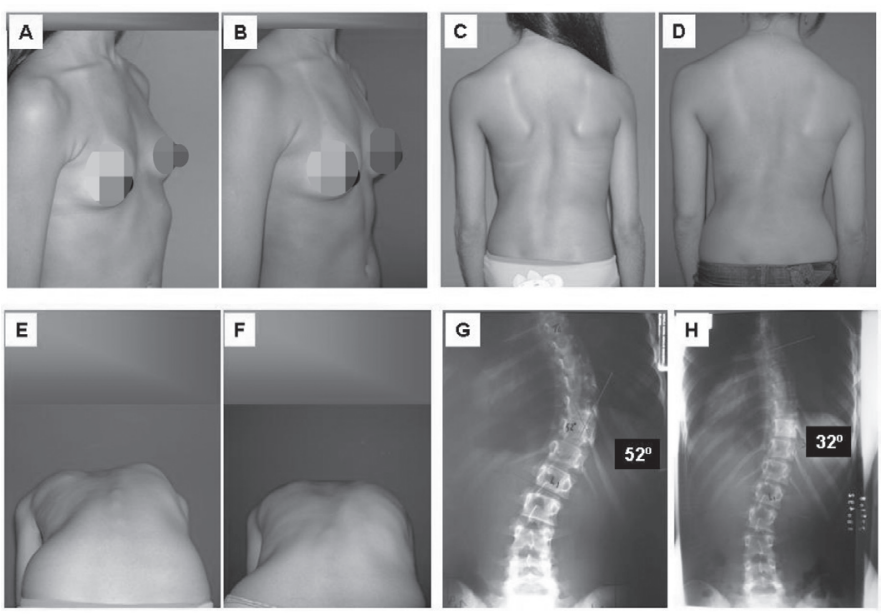

Figura 4 - Paciente do sexo feminino, 13 anos de idade, portadora de severa escoliose toracolombar dextroconvexa, de $52^{\circ}$ com limites em T6 e L1, Risser 1 e discreto PCL na parte anterior de seu tórax, associado a saliências moderadas dos rebordos costais (A, C, E, G). Ela já havia recebido indicação cirúrgica devido à gravidade da curva. Optou por tentar primeiro o tratamento concomitante com as órteses CDT e colete mostrados na figura 8. Executou com regularidade os exercícios do método DR, sem retirar as órteses CDT, diariamente. Utilizou as órteses CDT cerca de quatro horas por dia e o colete cerca de 19 horas por dia. Ela recebeu ao longo do tratamento três coletes novos, feitos por molde gessado. A melhora clínica 33 meses após início do tratamento, mostrada em $\mathrm{B}, \mathrm{D}, \mathrm{F}$, e a comprovação radiológica de redução da curva para $32^{\circ}$, mostrada em $\mathrm{H}$, afastaram a indicação cirúrgica.

\section{CONDUTAS MÉDICAS PARA SUCESSO DO MÉTODO DR}

O método DR requer critérios e supervisão médica adequada - ver resumo de nosso protocolo básico de condutas (Anexo 1). A supervisão médica deve ser prolongada, pois pode levar um ou mais anos para que uma correção se estabilize, dependendo de como o paciente execute o tratamento ao longo do tempo. O tratamento com órteses CDT I e/ou CDT II deve ser iniciado de acordo com a idade e o tipo de deformidade, conforme anteriormente detalhado. As órteses devem ser construídas de forma individualizada, de acordo com molde gessado e prescrição médica detalhada, com o formato das almofadas marcado no molde e suas medidas especificadas em prescrição médica. As hastes anterior e posterior devem ser de alumínio para permitir ajustes com a progressão do tratamento, pois o paciente cresce e/ou tem a forma de seu tórax modificada pelo tratamento. As hastes laterais devem ser feitas por pequenos tubos com porcas na sua extremidade anterior. Parafusos que sejam rosqueados nesses tubos devem ser sempre utilizados como mecanismo de compressão, pois seu apertar gradual, à medida que as estruturas osteocartilaginosas cedem, confere dinamicidade ao tratamento. Os exercícios complementam a dinâmica do tratamento, trabalhando os movimentos da caixa torácica e promovendo pressão interna em áreas deprimidas da parede torácica anterior. Instruções prévias sobre o método DR devem ser fornecidas ao paciente e seus responsáveis, comparando-o ao tratamento ortodôntico, ou seja, a um tratamento que deve ser prolongado, para remodelação gradativa da caixa torácica, visando estabilização da correção e ausência de riscos de recidiva. O paciente deve ser instruído a, nos primeiros meses (dois a quatro meses para o carinatum e seis a 12 meses para o excavatum), usar a órtese continuamente, inclusive para dormir, só retirando-a para o banho e a natação. Tal orientação é muito importante, inclusive para que o paciente se adapte à presença da órtese, pois, quanto maior o período sem a órtese, maior o incômodo em sua recolocação e menor a aceitação de continuidade do tratamento.

A compressão ideal pelo aperto dos parafusos, até que a órtese fique firme no tórax do paciente, deve ser determinada pelo médico no ato da consulta de colocação da mesma. O paciente deve ser instruído a afrouxar, mas não retirar a órtese, em caso de incômodo ou dor. Após alívio dos sintomas, ele deve ser instruído a aper-

\section{Anexo 1}

\section{PROTOCOLO Método DR}

1) Indicação correta de órtese CDT I e/ou CDT II, de acordo com a idade e o tipo de deformidade, sempre com parafusos como meio de compressão.

2) Órteses individualizadas, feitas por molde gessado e prescrição médica detalhada.

3) Conscientização do paciente sobre tratamento prolongado; êxito depende da execução das instruções; uso contínuo da(s) órtese(s) nos primeiros meses: exercícios diariamente ou por, pelo menos, cinco dias na semana.

4) Aperto dos parafusos controlado pelo médico nas consultas de colocação da órtese e de acompanhamento; evitar hipercorreção; instituir órtese adicional, se necessário.

5) O acompanhamento médico deve ser feito a cada dois ou três meses no primeiro ano e a cada quatro meses a seguir.

6) Ajustes na órtese; acompanhar crescimento e modificações da caixa torácica com o tratamento; eventual construção de nova órtese.

7) Prescrição de exercícios, sempre com a órtese no corpo; ensinados por fisioterapeuta treinado; não exagerar na carga em academias.

8) Prevenir irritação cutânea intensa: usar roupa justa de tecido de algodão sob a órtese ou capas limpas de malha de algodão nas almofadas; controlar hipercorreção.

9) Fotografias clínicas obtidas sempre de um mesmo ângulo, antes e durante o tratamento.

10) Coordenação pelo médico de processo terapêutico que envolve participação de outros profissionais da saúde, como técnico em órteses e fisioterapeuta. 
tar novamente os parafusos até o grau anteriormente determinado. O progresso no grau de aperto e compressão deve acontecer de acordo com a tolerância do paciente. Raramente, é indicada medicação para controle de dor. O acompanhamento médico deve ser feito a cada dois ou três meses no primeiro ano e a cada quatro meses a seguir. Eventualmente, a construção de nova órtese por novo molde gessado pode ser necessária. Um dos ajustes frequentemente necessários à medida que o paciente cresce e seu tórax se altera com o tratamento é o alargamento látero-lateral. Tal alargamento faz-se pela abertura dos ângulos da haste posterior e também da haste anterior. A diminuição do comprimento dos parafusos e das hastes laterais ou adiantamento da dobra dos ângulos da haste anterior podem ser necessários para possibilitar compressão adicional. Muitas vezes ocorre encurvamento na parte mediana das hastes anterior e/ou posterior, tornando-se necessário que o médico prescreva retificação dessas peças, além de duplicação das mesmas para reforço, para que a compressão volte a ser eficiente. Tais modificações e ajustes proporcionam possibilidade de aperto adicional a parafusos que já haviam penetrado por completo nas hastes laterais. Pode-se também fazer necessária alteração no posicionamento, no tamanho e na forma das almofadas.

A prescrição de exercícios com a órtese no corpo é fundamental. Os exercícios devem ser ensinados e listados por fisioterapeuta treinado, e o paciente deve realizá-los diariamente. Exercícios em aparelho peck deck (crucifixo) em academias também devem ser prescritos e feitos três vezes na semana, sempre com o paciente em uso da(s) órtese(s) CDT. A prescrição médica deve enfatizar que tais exercícios, feitos sob a supervisão de educador físico, têm como objetivo não a hipertrofia muscular, mas sim a movimentação da caixa torácica, e que, portanto, a carga dos exercícios não deve ser exagerada, devendo-se priorizar um programa que aumente prioritariamente e de maneira gradativa não a carga, mas sim o número de repetições, de acordo com a idade do paciente. Musculação, natação ou outros exercícios isoladamente não corrigem uma deformidade pectus. Podem apenas eventualmente disfarçá-la pela hipertrofia muscular. A prática de musculação sem a utilização concomitante de uma órtese CDT pode agravar uma protrusão torácica e contribuir para seu enrijecimento. Uma depressão esternal também pode tornar-se mais rígida e ter seu aspecto agravado se houver desenvolvimento excessivo dos peitorais. A remodelação corretiva do pectus excavatum, portanto, só acontece quando uma compressão dos rebordos costais potencializa o aumento da pressão intratorácica provocada pelos exercícios. A natação, entretanto, deve ser prescrita, sem a órtese, em qualquer idade, nos estilos costas e livre - ou crawl -, visando a manutenção da maleabilidade torácica e a melhora do condicionamento físico, com consequente melhor postura do tronco, ajudando a combater a má postura, que pode agravar a deformidade pectus.

$\mathrm{O}$ paciente deve ser instruído a, no dia em que for à academia, realizar os exercícios aprendidos e, no dia em que não for, realizá-los em casa, sempre com a órtese no corpo. Para minimizar a irritação cutânea, usar roupa justa de tecido de algodão sob a órtese ou fazer capas de malha de algodão com elástico nas bordas, vesti-las nas almofadas e trocá-las diariamente por outras limpas. A irritação cutânea e eventual hipercorreção são controladas pelo acompanhamento médico adequado, sem necessidade de interrupção do tratamento. Raramente há necessidade de uso de medicação tópica e, quando isso acontece, o paciente deve ser instruído a ficar duas horas sem a órtese, retirando a oleosidade de creme ou pomada com álcool antes de recolocá-la. Haje e Haje descreveram as condutas a serem adotadas para controle da hipercorreção ${ }^{(33)}$. Fotografias clínicas devem ser feitas, sempre de um mesmo ângulo, antes, durante e depois do tratamento, abrangendo exclusivamente o tronco do paciente. O arquivamento apropriado dessas fotos clínicas documenta a evolução do caso, dando suporte para interpretação e conduta clínica, além de servir de estímulo para o paciente seguir adiante com o tratamento. O tratamento deve ser feito por um trabalho de equipe composta por médico, técnico em órtese e fisioterapeuta, com o médico coordenando todo o processo.

De acordo com Haje, portadores de pectus podem ter escolioses discretas e flexíveis e cifoses posturais melhoradas apenas pelo método $\mathrm{DR}^{(23)}$. Nossos achados sugerem que escolioses mais graves, entre $20^{\circ} \mathrm{e}$ $52^{\circ}$, quando associadas a deformidades pectus, possam beneficiar-se da associação de tratamento concomitante de colete e método DR, mas isso necessita maiores estudos para comprovação futura. O presente estudo não visou pormenorizar os achados referentes à etiologia $\mathrm{e}$ à patogênese e, muito menos, analisar detalhadamente os achados dos exames de imagem e dos resultados do tratamento concomitante de escolioses. Visa sim, proporcionar a transmissão da experiência dos autores na abordagem das deformidades pectus, abrindo caminhos 
para novas pesquisas no assunto e tornando o tratamento que preconizam executável por todos os médicos interessados e, em especial, os ortopedistas.

\section{CONSIDERAÇÕES FINAIS}

Nossos resultados quanto à etiologia e à patogênese são altamente sugestivos de distúrbios na formação, crescimento e desenvolvimento das estruturas osteocartilaginosas da parede torácica anterior, demonstrando o vínculo das deformidades pectus com a ortopedia. A opção de tratar conservadoramente as deformidades pectus tem como base os princípios de Nicolas Andry, considerado "o pai da ortopedia", e os efeitos desse tratamento podem ser explicados pela lei da remodelação óssea de Julius Wolff. Forças terapêuticas aplicadas regularmente sobre ossos e cartilagens deformados podem produzir uma remodelação gradual no sentido benéfico, corretivo, e isso pode ser especialmente observado na parede torácica anterior, uma região maleável.

\section{REFERÊNCIAS}

1. Garcia VF, Seyfer AE, Graeber GM. Reconstruction of congenital chest-wall deformities. Surg Clin North Am. 1989;69(5):1103-18.

2. Haje DP, Haje SA, Simioni MA. Prevalência das deformidades pectus carinatum e pectus excavatum em escolares do Distrito Federal. Brasília Med. 2002;39(1/4):10-5.

3. Einsiedel E, Clausner A. Funnel chest. Psychological and psychosomatic aspects in children, youngsters and young adults. J Cardiovasc Surg (Torino). 1999;40(5):733-6.

4. Haje SA, Haje DP. Tratamento conservador e reabilitação de pacientes com deformidades pectus: uma experiência de 29 anos. Med Reabil. 2007;26(1):1-6.

5. Bryson V. Development of the Sternum in Screw Tail Mice. Anat Rec. 1945;91:119-41.

6. Herdner M. Le Sternum de l'enfant. Etude radiologique das anomalies de son developpement. Rev Orthop. 1947;33(6):475-93.

7. Currarino G, Silverman N. Premature obliteration of the sternum sutures and pigeon breast deformity. Radiolo!y. 1958; 70(4):352-40.

8. Gabrielsen TO, Ladyman GH. Early closure of the sternal sutures and congenital heart disease. Am J Roentgenol Radium Ther Nucl Med. 1963;89:975-83.

9. Fischer KC, White RI Jr, Jordan CE, Dorst JP, Neil CA. Sternal abnormalities in patients with congenital heart disease. Am J Roentgenol Radium Ther Nucl Med. 1973;119(3):530-8.

10. Ravitch MM. Congenital deformities of the chest wall and their operative correction. Philadelphia: WB Saunders; 1977.

11. Haje SA, Raymundo JLP. Considerações sobre deformidades da parede torácica anterior e apresentação de tratamento conservador para as formas com componentes de protrusão. Rev Bras Ortop. 1979;14(4):167-78.

12. Kuhn JP. The Thorax. In: Silverman FN. Editor. Caffey's pediatric X-ray diagnosis. Chicago: Year Book Medical Publishers;1985. p.1097-9.

13. Haje SA, Antunes EJ, Raymundo JLP, Dourado JN. Pectus carinatum: enfoque atual. Rev Bras Ortop. 1988;23(9):257-64.

14. Lees RF, Caldicott JH. Sternal anomalies and congenital heart disease. Am J Roentgenol. 1975;124(3):423-7.

15. Ogden JA, Conlogue GJ, Bronson ML, Jensen PS. Radiology of postnatal skeletal development. II.The manubrium and sternum. Skeletal Radiol. 1979;4(4):189-95.

16. Wong M, Carter DR. Mechanical stress and morphogenetic endochondral ossification of the sternum. J Bone Joint Surg Am. 1988; 70(7):992-1000.

17. Shimomura $Y$, Wezeman FH, Ray RD. The growth cartilage plate of the rat rib: cellular differentiation. Clin Orthop Relat Res. 1973;(90):246-54

18. Yamasaki K, Inui S. Lesions of articular, sternal and growth plate cartilage in rats. Vet Pathol. 1985;22(1):46-58
O tratamento das deformidades pectus implica compreender conceitos ortopédicos básicos. Portanto, a natureza das deformidades pectus é relacionada à ortopedia e o seu estudo deve fazer parte do currículo de assuntos ortopédicos. A simples prescrição de uma órtese não significa tratar uma deformidade pectus. Um protocolo de condutas deve ser obedecido para um tratamento correto e bem-sucedido.

\section{AGRADECIMENTOS}

Os autores agradecem aos médicos Moacir Silva Neto, Guilherme de Souza e Cássia, Gustavo Reis Alves de Oliveira, Rodrigo Coimbra Batista e Tito Lívio Mundim, pelo auxílio na interpretação das tomografias computadorizadas multislices demonstradas neste trabalho. Agradecem também ao colega Rubens Paiva Souza, pelo auxílio no levantamento dos casos de portadores de pectus e escoliose que receberam o tratamento concomitante.

19. Haje SA, Bowen JR. Preliminary results of orthotic treatment of pectus deformities in children and adolescents. J Pediatr Orthop. 1992;12(6):795-800.

20. Haje SA. latrogenic pectus carinatum: a case report. Int Orthop (SICOT). 1995; 19(6):370-73.

21. Haje SA, Bowen JR, Harcke HT, Guttenberg ME, Bacon CR. Disorders in the sternum growth and pectus deformities: an experimental model and clinical correlation. Acta Ortop Bras. 1998; 6(2):67-75.

22. Haje SA, Harcke HT, Bowen JR. Growth disturbance of the sternum and pectus deformities: imaging studies and clinical correlation. Pediatr Radiol. 1999; 29(5):334-41.

23. Haje SA. Tórax e cintura escapular. In: Hebert SK, Xavier R, Pardini Junior AG, Barros Filho TEP.Ortopedia e traumatologia: princípios e prática. $3^{a}$ ed. Porto Alegre: Artmed; 2003. p.161-84.

24. Haje SA, Haje DP. Tórax e cintura escapular. In: Hebert SK, Barros Filho TEP, Xavier R, Pardini Junior AG. Ortopedia e traumatologia: princípios e prática. $4^{a}$ ed. Porto Alegre: Artmed. 2009; p.147-65.

25. Haje SA. Deformidades pectus: novos conceitos e abordagem ortopédica em crianças e adolescentes - $1^{\text {a }}$ parte. Rev Bras Ortop. 1995;30(1/2):75-9.

26. Beirão ME. Tratamento conservador do pectus carinatum com uso de órtese. Rev Bras Ortop. 1999;34(11/12): 575-8.

27. Egan CJ, Du Bois JJ, Morphy M, Samples, TL, Lindell B. Compressive orthotics in the treatment of asymetric pectus carinatum: a preliminary report with an objective radiographic marker. J Pediatr Surg. 2000;35(8):1183-6.

28. Frey AS, Garcia VF, Brown RL, Inge TH, Ryckman FC, Cohen AP, et al. Nonoperative management of pectus carinatum. J Pediatr Surg. 2006;41(1):40-5

29. Banever GT, Konefal SH, Gettens K, Moriarty KP. Nonoperative correction of pectus carinatum with orthotic bracing. J Laparoendosc Adv Surg Tech. 2006;16(2):164-7.

30. Kravarussic D, Dicken BJ, Dewar R, Harder J, Poncet P, Schneider M, et al. The Calgary protocol for bracing of pectus carinatum: a preliminary report. J Pediatr Surg. 2006;41(5):923-6.

31. Martinez-Ferro M, Fraire C, Bernard S. Dynamic compression system for the correction of pectus carinatum. Semin Pediatr Surg. 2008;17(3):194-200.

32. Haje SA. Deformidades pectus: novos conceitos e abordagem ortopédica em crianças e adolescentes - $2^{\mathrm{a}}$ parte. Rev Bras Ortop. 1995;30(3):143-9.

33. Haje SA, Haje DP. Overcorrection during treatment of pectus deformities with DCC orthoses: experience in 17 cases. Int Orthop (SICOT). 2006;30(4):262-7.

34. Haje SA, Haje DP, Guerra JB, Petrenko JR. Órtese inclinada de uso contínuo e exercícios para tratamento da escoliose idiopática: uma nova proposta. Brasília Med. 2008;45(1):10-20 